gap $>\mathrm{g}:=$ SymmetricGroup $(4)$;

$\operatorname{Sym}\left(\left[\begin{array}{lll}1 & 4\end{array}\right]\right)$

gap> tbl:= CharacterTable ( $\mathrm{g}$ ); ; HasIrr ( tbl );

false

01234 gap> tblmod2:= CharacterTable ( tbl, 2 );

$05=$ total: 1413144

BrauerTable( $\operatorname{Sym}([1 \ldots 4]), 2$ )

1: 2242 gap> tblmod2 = CharacterTable $(t b 1,2$ );

2: 256 .

Journal of Software for

01234 gap> libtbl:= CharacterTable( "M" );

$06=$ total: $1{ }^{4} 13144$ CharacterTable( "M")

fail $\quad r i n g ~ r 1=32003,(x, y, z), d s$;

gap> CharacterTable ( "Symmetric", 4 ) ;int a, b, c, t=11, 5, 3,0;

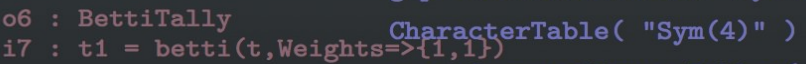

gap> ComputedBrauerTables ( tbl );

$\begin{array}{lllll}0 & 1 & 2 & 3 & 4\end{array}$

$07=$ total: $14 \begin{array}{llll}1 & 4 & 14 & 4\end{array}$

$0: 1 . .$.

[ BrauerTable( $\operatorname{Sym}([1 \ldots 4]), 2$ option(noprot);

poly $f=x^{\wedge} a+y^{\wedge} b+z^{\wedge}(3 * c)+x^{\wedge}(c+2) * y^{\wedge}(c-1)+x^{\wedge}$ $x^{\wedge}(c-2) * y^{\wedge} c *\left(y^{\wedge} 2+t * x\right) \sim 2$;

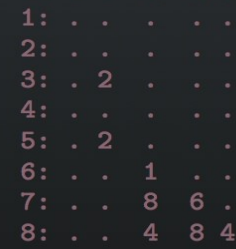

o7 : BettiTally

i8 : peek t1

timer=1;

ring $r 2=32003,(x, y, z), d p$;

poly $f=i m a p(r 1, f)$;

ideal $j=j a c o b(f)$;

$\operatorname{vdim}(\operatorname{std}(j))$;

$\Rightarrow 536$

$\operatorname{vdim}(\operatorname{std}(j+f))$;

$\Longrightarrow 195$

timer $=0$; // reset timer

$08=\operatorname{BettiTally}\{(0,\{0,0\}, 0) \Rightarrow 1\}$

$(1,\{2,2\}, 4) \Rightarrow 2$

$(1,\{3,3\}, 6) \Rightarrow 2$

$(2,\{3,7\}, 10) \Rightarrow 2$

$(2,\{4,4\}, 8) \Rightarrow 1$

$(2,\{4,5\}, 9) \Rightarrow 4$

$(2,\{5,4\}, 9) \Rightarrow 4$

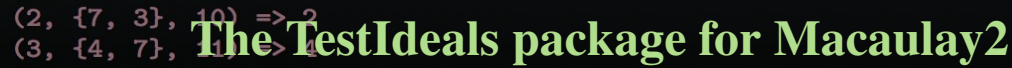

$(3,\{5,5\}, 10) \Rightarrow 6$

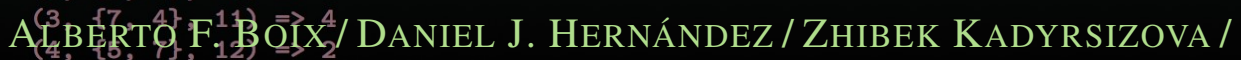

(4.MORAECEPAF KATZMAN / S ARA MALEC / MARCUS ROBINSON /

Karl SchWede / DANiEL SMolkin / Pedro Teixeira / Emily E. WitT 


\title{
The TestIdeals package for Macaulay2
}

\author{
Alberto F. Boix, Daniel J. Hernández, Zhibek Kadyrsizova, \\ Mordechai Katzman, Sara Malec, Marcus Robinson, \\ Karl Schwede, Daniel Smolkin, Pedro Teixeira and Emily E. Witt
}

\begin{abstract}
We describe a Macaulay2 package for computations in prime characteristic commutative algebra. This includes those for Frobenius powers and roots, $p^{-e}$-linear and $p^{e}$-linear maps, singularities defined in terms of these maps, different types of test ideals and modules, and ideals compatible with a given $p^{-e}$-linear map.
\end{abstract}

1. IntRODUCTION. This paper describes methods for computing objects and numerical invariants in prime characteristic commutative algebra, implemented in the package TestIdeals for the computer algebra system [Macaulay2]. A ring $R$ of prime characteristic $p$ comes equipped with the Frobenius endomorphism

$$
F: R \rightarrow R \text { given by } F(x)=x^{p},
$$

which is the basis for many constructions and methods. Notably, the Frobenius endomorphism can be used to detect whether a ring is regular [Kunz 1969], and further, to quantify how far a ring is from being regular, measuring the severity of a singularity.

In this direction, two notable applications of the Frobenius endomorphism are the theory of tight closure (see [Hochster and Huneke 1990; Hochster 2007] for an introduction) and the resulting theory of test ideals (see the survey [Schwede and Tucker 2012]). These methods are used by a wide group of commutative algebraists and algebraic geometers.

The TestIdeals package was started during a Macaulay2 development workshop in 2012, hosted by Wake Forest University. The package, at that time called PosChar, aimed to provide a unified and efficient set of tools to study singularities in characteristic $p>0$, and in particular, collecting and implementing several algorithms that had been described in research papers. Since then, PosChar has been split into two

A.F. Boix was supported by Israel Science Foundation (grant No. 844/14) and Spanish Ministerio de Economía y Competitividad MTM2016-7881-P. D. J. Hernández was partially supported by NSF DMS \#1600702. Z. Kadyrsizova was partially supported by NSF DMS \#1401384 and the Barbour Scholarship at the University of Michigan. K. Schwede was supported by NSF Career Grant DMS \#1252860/1501102, NSF FRG Grant DMS \#1265261/1501115, NSF grant \#1801849 and a Sloan Fellowship. D. Smolkin was supported by NSF RTG Grant DMS \#1246989, NSF Career Grant DMS \#1252860/1501102, and NSF FRG Grant DMS \#1265261/1501115. E.E. Witt was partially supported by NSF DMS \#1623035.

MSC2010: primary 13A35, 14F18; secondary 14J17.

Keywords: Macaulay2, Test ideals, F-regular, F-rational, F-pure, F-injective, Frobenius root, compatibly split ideals.

TestIdeals version 1.01 
packages, TestIdeals and [FrobeniusThresholds] (see [Hernández et al. 2019]), and much more functionality has been added by many contributors, during several more Macaulay2 development workshops. ${ }^{1}$

Starting at least with Kunz [1969] and Fedder [1983], it has been known that the Frobenius endomorphism offers effective methods for measuring singularities in positive characteristic. However, the algorithms that form the basis of this package are the methods for computing Frobenius roots, $\star-$ closures, and parameter test ideals. These first appeared in [Katzman 2008; 2010; Blickle et al. 2008; 2009]. Another algorithm in [Katzman and Schwede 2012] for computing prime ideals compatible with a given $p^{-e}$-linear map was implemented and used to produce the examples in that paper. The methods for computing test ideals and test modules that were used implicitly in papers such as [Blickle et al. 2010; Katzman et al. 2009; Schwede and Tucker 2014a] became implementable via the Frobenius roots functionality. Algorithms for computing $F$-pure thresholds from [Hernández 2014; 2015; Hernández and Teixeira 2017] form some of the key methods in the forthcoming FrobeniusThresholds package.

2. Frobenius powers AND FrobeniUs RoOts. Let $R$ denote a commutative ring of prime characteristic $p$.

Definition 2.1. Given an ideal $I \subseteq R$ and an integer $e \geq 0$, we define the $p^{e}$-th Frobenius power of $I$, denoted $I^{\left[p^{e}\right]}$, to be the ideal generated by the $p^{e}$-th powers of all elements of $I$.

It is easy to see that if $I$ is generated by $g_{1}, \ldots, g_{\ell}$, then $I^{\left[p^{e}\right]}$ is generated by $g_{1}^{p^{e}}, \ldots, g_{\ell}^{p^{e}}$.

Definition 2.2. Given an ideal $I \subseteq R$ and an integer $e \geq 0$, we define the $p^{e}$-th Frobenius root of $I$, denoted $I^{\left[1 / p^{e}\right]}$, to be the smallest ideal $J$ such that $I \subseteq J^{\left[p^{e}\right]}$, if such an ideal exists.

Frobenius roots always exist in polynomial and power series rings, and in $F$-finite regular rings (see [Blickle et al. 2008, §2] and [Katzman 2008, §5]). Below is an example of a computation of a Frobenius root in a polynomial ring. In Section $2 \mathrm{~A}$ we describe the main ideas behind such computations.

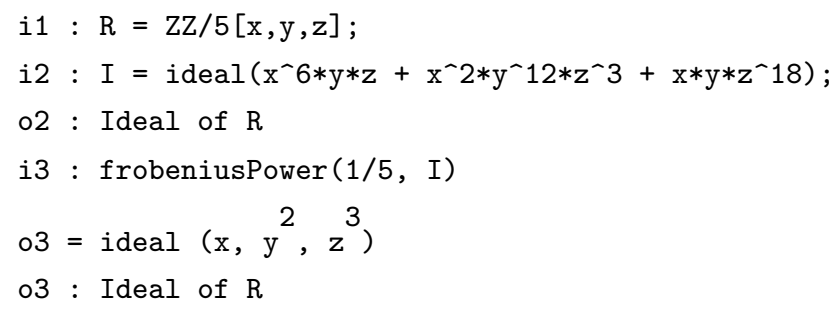

2A. The mathematics behind the computation of Frobenius roots. We can also describe Frobenius roots as follows: In a (sufficiently local) regular ring, we have an identification of $R$ with its canonical module $\omega_{R}$. On the other hand, the Grothendieck dual to the $e$-iterated Frobenius map ${ }^{2} R \rightarrow R^{1 / p^{e}}$

\footnotetext{
${ }^{1}$ Development workshops hosted by the University of California, Berkeley $(2014,2017)$, Boise State University (2015), and the University of Utah (2016).

${ }^{2}$ In a reduced ring $R$, it is often convenient to express the Frobenius endomorphism $R \rightarrow R$ as the inclusion $R \hookrightarrow R^{1 / p}$, so that the source and target are distinguished.
} 
provides a map

$$
T: \omega_{R^{1 / p^{e}}} \rightarrow \omega_{R}
$$

which, using the identification $R \cong \omega_{R}$, gives us a map

$$
T: R^{1 / p^{e}} \rightarrow R
$$

It is not difficult to prove that $T\left(I^{1 / p^{e}}\right)=I^{\left[1 / p^{e}\right]}$, where $I^{1 / p^{e}} \subseteq R^{1 / p^{e}}$ is simply the ideal of $p^{e}$-th roots of the elements of $I$. In the case that

$$
R=\mathbb{K}\left[x_{1}, \ldots, x_{d}\right],
$$

where $\mathbb{K}$ is a perfect field, $R^{1 / p^{e}}$ is a free $R$-module with basis consisting of the monomials

$$
\boldsymbol{x}^{\lambda / p^{e}}=x_{1}^{\lambda_{1} / p^{e}} \cdots x_{d}^{\lambda_{d} / p^{e}},
$$

where $0 \leq \lambda_{i} \leq p^{e}-1$. Furthermore, the map $T: R^{1 / p^{e}} \rightarrow R$ is simply the projection defined as follows:

$$
T\left(x_{1}^{\lambda_{1} / p^{e}} \cdots x_{d}^{\lambda_{d} / p^{e}}\right)= \begin{cases}1 & \text { if } \lambda_{i}=p^{e}-1 \text { for all } i \\ 0 & \text { otherwise }\end{cases}
$$

Using this, it is not difficult to see that if

$$
f^{1 / p^{e}}=\sum_{\lambda} f_{\lambda} x^{\lambda / p^{e}}
$$

where $\lambda$ runs over the tuples $\left(\lambda_{1}, \ldots, \lambda_{d}\right)$ with $0 \leq \lambda_{j} \leq p^{e}-1$, and $f_{\lambda} \in R$, then $(f)^{\left[1 / p^{e}\right]}=T\left((f)^{1 / p^{e}}\right)$ is the ideal generated by the $f_{\lambda}$. We can then compute $I^{\left[1 / p^{e}\right]}$ for more general $I$ by linearity: if $I=$ $\left(f_{1}, \ldots, f_{m}\right)$, then $I^{\left[1 / p^{e}\right]}=\sum\left(f_{i}\right)^{\left[1 / p^{e}\right]}$.

Complexity. The computation of Frobenius roots is the main component behind many of the methods in TestIdeals. Hence, it is important to understand how this is implemented, and its computational complexity.

The computation of Frobenius roots of ideals reduces to the case of principal ideals, and its complexity grows linearly with the number of generators of the ideal. Furthermore the calculation of $(f)^{\left[1 / p^{e}\right]}$ reduces to finding the terms in the right-hand side of (2-2), which essentially amounts to taking each term in $f$, computing the $p^{e}$-th root of its coefficient, and dividing the monomial exponent vector by $p^{e}$ with remainder. Hence the complexity of computing $(f)^{\left[1 / p^{e}\right]}$ is proportional to the number of terms in $f$, and is independent of its degree. We emphasize that the calculation of Frobenius roots does not involve the calculation of Gröbner bases.

2B. Dual to Frobenius on quotient rings and Frobenius on top local cohomology. For any reduced ring $R$ with finite $e$-iterated Frobenius map identified with $R \hookrightarrow R^{1 / p^{e}}$, we always have a map

$$
T_{R}: \omega_{R^{1 / p^{e}}} \rightarrow \omega_{R} .
$$


At a maximal ideal $\mathfrak{m}$ of height $d$, this map is Matlis dual to the Frobenius map on $H_{\mathfrak{m}}^{d}(R)$. Hence we can study local cohomology by studying this map. If $R=S / I$, where $S$ is a polynomial ring over a finite field, we can implement this map [Fedder 1983; Katzman 2008]. We briefly explain the case of a hypersurface here.

If $I=(f)$, again $\omega_{R} \cong R=S / I$, and the action of $T_{R}$ can be computed on $S$. In fact, if $\bar{J} \subseteq R \cong \omega_{R}$ has preimage $J \subseteq S$, then setting $u=f^{p^{e}-1}$, we have that $T_{R}\left(\bar{J}^{1 / p^{e}}\right)$ is the image of $(u J)^{\left[1 / p^{e}\right]}$ in $R$. More generally, for non-hypersurfaces, the analog to $u$ is chosen to be an element that maps onto the generator of the cyclic $R$-module

$$
\frac{\left(I^{\left[p^{e}\right]}: I\right) \cap\left(\Omega^{\left[p^{e}\right]}: \Omega\right)}{I^{\left[p^{e}\right]}},
$$

where $\Omega$ is an ideal of $S$ with $I \subseteq \Omega$ and whose image in $R$ is isomorphic to the canonical module of $R$; see [Katzman 2008] for details. Here we compute two examples.

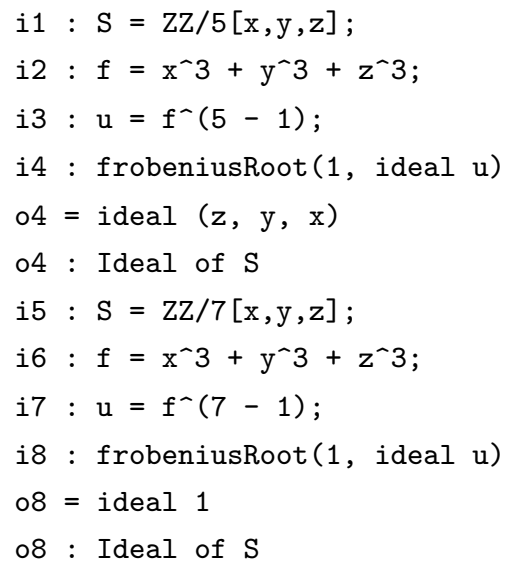

The above example has shown that the Frobenius map on top local cohomology of the cone over the Fermat elliptic curve is injective in characteristic 7 (the dual map $\omega_{R^{1 / p}} \rightarrow \omega_{R}$ is surjective) and not injective in characteristic 5 (the dual map $\omega_{R^{1 / p}} \rightarrow \omega_{R}$ is not surjective).

2C. A generalization of Frobenius powers and roots. Let $R$ be an $F$-finite regular ring. We can extend the definition of Frobenius powers as follows.

Definition 2.3 [Hernández et al. 2018]. Let $I \subseteq R$ be an ideal.

(a) If $n$ is a positive integer with base $p$ expansion $n=d_{0}+d_{1} p+\cdots+d_{r} p^{r}$, we define

$$
I^{[n]}=I^{d_{0}}\left(I^{d_{1}}\right)^{[p]} \cdots\left(I^{d_{r}}\right)^{\left[p^{r}\right]} .
$$

(b) If $t$ is a nonnegative rational number of the form $t=a / p^{e}$, we define $I^{[t]}=\left(I^{[a]}\right)^{\left[1 / p^{e}\right]}$.

(c) If $t$ is any nonnegative rational number, and $\left\{a_{n} / p^{e_{n}}\right\}_{n \geq 1}$ is a sequence of rational numbers converging to $t$ from above, we define $I^{[t]}$ to be the stable value of the nondecreasing chain of ideals $\left\{I^{\left[a_{n} / p^{e_{n}}\right]}\right\}_{n \geq 1}$. 


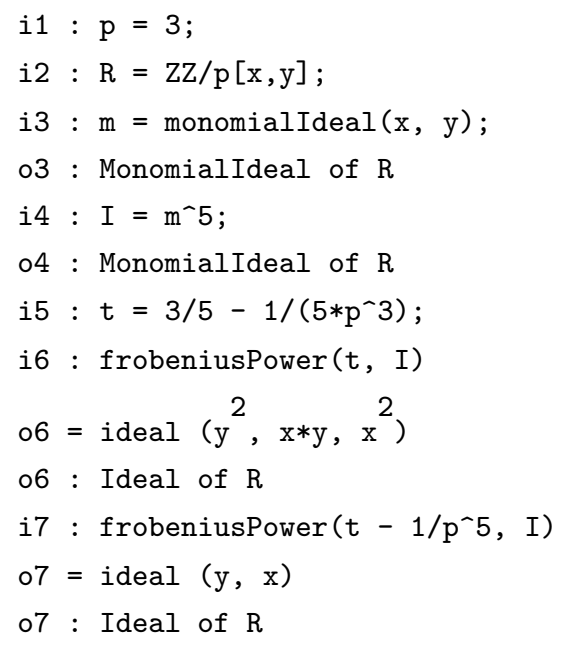

The generalized Frobenius powers of an ideal are test ideals (see Section 4) of sufficiently general linear combinations of generators of that ideal. Thus, the ideals computed above are test ideals of generic quintics in two variables in characteristic $p=3$, and these computations suggest that $3 / 5-1 /\left(5 p^{3}\right)$ is a higher $F$-jumping exponent of such quintics, which is indeed the case for any $p \equiv 3 \bmod 5$ (see [Hernández et al. 2020]). To illustrate, compare the computations above with the following.

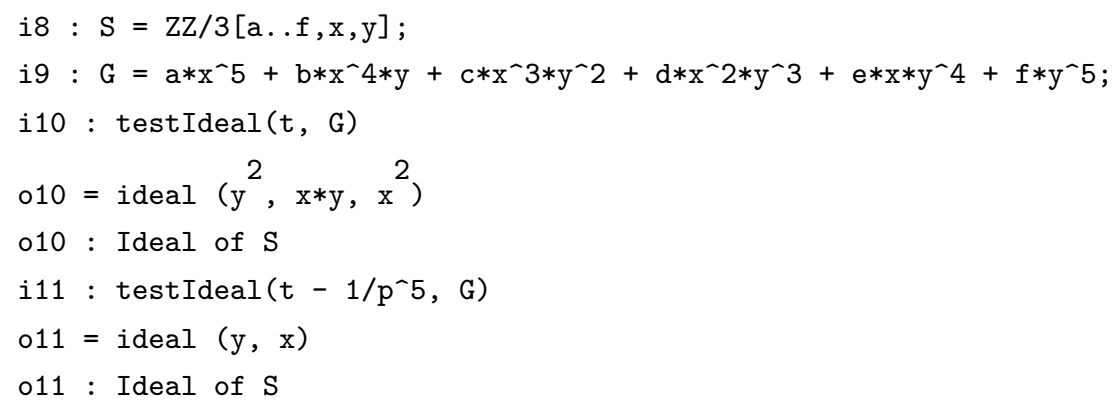

Caveat. The computations of Frobenius powers with exponents whose denominators are not powers of the characteristic $p$ are often very slow, and in several instances it is more efficient to introduce more variables and compute the test ideal of a "very general" linear combination of the generators of the ideal, as above. The code used for computations of Frobenius powers needs to undergo significant optimization.

2D. Frobenius roots of submodules of free modules. Let $S$ be a polynomial ring or power series ring. Given a submodule $M$ of the free module $S^{k}$, there is a smallest submodule $N$ of $S^{k}$ that contains $M$, for which $M \subseteq N^{\left[p^{e}\right]}$. Here, $N^{\left[p^{e}\right]}$ is the submodule of $S^{k}$ generated by the vectors of $N$ with all coordinates raised to the $p^{e}$-th power. (See [Katzman and Zhang 2014].) Extending the previous definitions, we call this $N$ the $p^{e}$-th Frobenius root of $M$, and denote it by $M^{\left[1 / p^{e}\right]}$.

Example 2.4. Let $R=\mathbb{Z} / p \mathbb{Z}[a, b, c, d]$, and consider the ideals $\mathfrak{m}=(a, b, c, d)$ and

$$
I=(a, b) \cap(a, c) \cap(c, d) \cap\left(c+d, a^{3}+b d^{2}\right) .
$$


Then $R / I$ is a 3-dimensional non-Cohen-Macaulay ring. Matlis duality applied to $H_{\mathfrak{m}}^{2}(R / I)$ with its natural Frobenius map yields a $p^{-1}$-linear map $U$ on $\operatorname{Ext}_{R}^{2}(R / I, R)$.

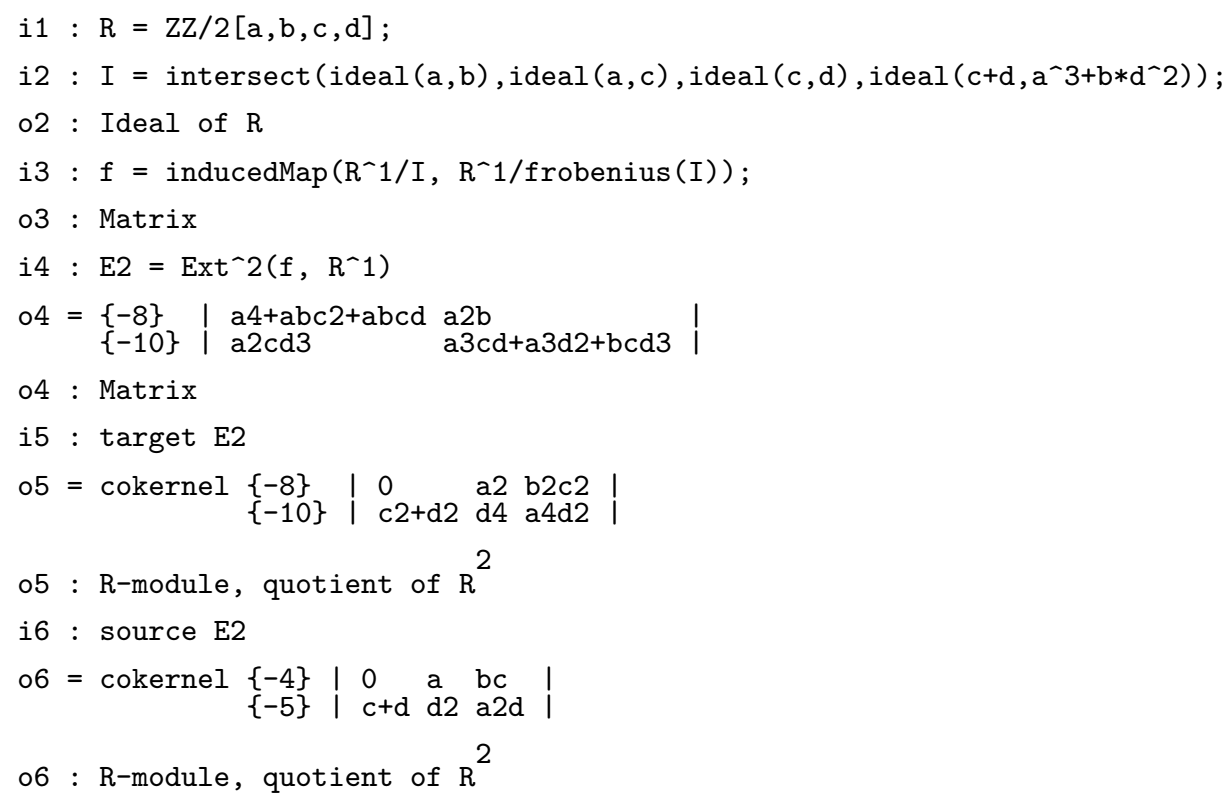

The Frobenius map on $H_{\mathfrak{m}}^{2}(R / I)$ is injective if and only if the image of (Image $\left.U\right)^{[1 / p]}$ in $\operatorname{Ext}_{R}^{2}(R / I, R)$ as computed above is the whole of $R^{2}$.

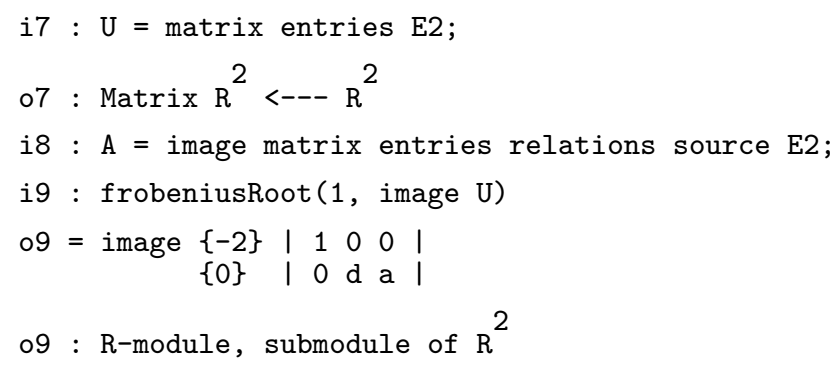

The calculation above shows that $R / I$ is not $F$-injective (see Section 3). Moreover, it shows that $H_{\mathfrak{m}}^{2}(R / I) \cong \operatorname{Ann}_{E^{2}} A^{t}$, where $E$ is the injective hull of $R_{\mathfrak{m}} / \mathfrak{m} R_{\mathfrak{m}}$, and the Frobenius map on $H_{\mathfrak{m}}^{2}(R / I)$ induced from Frobenius on $R$ is given by $U^{t} \Theta$, where $\Theta$ is the induced Frobenius on $E$. The submodule of nilpotent elements in $H_{\mathfrak{m}}^{2}(R / I)$ is given by $\operatorname{Ann}_{E^{2}} B^{t}$, where $B$ is the smallest submodule of $R^{2}$ containing Image $A+$ Image $U$ such that $U B \subseteq B^{[p]}$. The method ascendModule can be used to calculate $B$ (see a detailed description of the similar method ascendIdeal in Section 4).

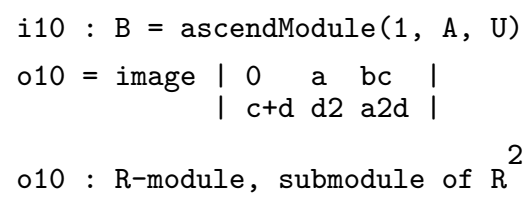


3. F-SingularitiES. The TestIdeals package includes methods for determining if a ring is $F$-injective, $F$-pure, $F$-rational, or $F$-regular.

\section{A. F-injectivity.}

Definition 3.1. A local ring $(R, \mathfrak{m})$ is called $F$-injective if the map $H_{\mathfrak{m}}^{i}(R) \rightarrow H_{\mathfrak{m}}^{i}\left(R^{1 / p}\right)$ is injective for all $i>0$. An arbitrary ring is called $F$-injective if its localization at each prime ideal of $R$ is $F$-injective.

The function isfInjective determines whether the ring $R=S / I$ is $F$-injective, where $S$ is a polynomial ring.

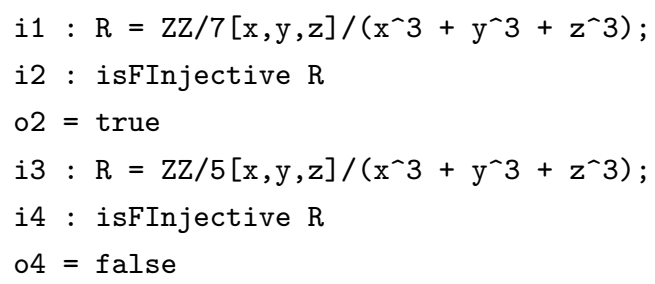

Equivalently, a ring is $F$-injective if the maps on the cohomology of the dualizing complex

$$
h^{-i} \omega_{R^{1 / p}}^{\bullet} \rightarrow h^{-i} \omega_{R}^{\bullet}
$$

surject for all $i$. Note that $h^{-i} \omega_{R}^{\bullet} \cong \operatorname{Ext}^{\operatorname{dim} S-i}(R, S)$, the latter of which, Macaulay2 readily computes. The algorithm used by isFInjective works by checking the surjectivity of the dual Frobenius map

$$
\operatorname{Ext}^{\operatorname{dim} S-i}\left(R^{1 / p}, S\right) \rightarrow \operatorname{Ext}^{\operatorname{dim} S-i}(R, S)
$$

We begin by computing the map $R \rightarrow R^{1 / p}$ using [PushForward]. Next, the algorithm computes $\operatorname{Ext}^{i}(\ldots, S)$ applied to the map from the previous step. Then $R$ is $F$-injective precisely when the cokernel of $\operatorname{Ext}^{i}(\ldots, S)$ is trivial for $i$.

The Frobenius action on top local cohomology (dual to $\omega_{R}$ ) is usually computed in a different (faster) way than the other cohomologies, and this is modified by the CanonicalStrategy option. The default value for this option is Katzman, which instead of using the PushForward package, relies on the fact that we already know how to compute the Frobenius action on the canonical module, as described in Section 2B.

The performance of the algorithm can be improved if the ring of interest is nice enough. If the ring is Cohen-Macaulay, then setting AssumeCM $\Rightarrow$ true (the default value is false) lets the algorithm check the Frobenius action only on top cohomology (which is typically much faster, as explained above). When studying a reduced ring, setting AssumeReduced $\Rightarrow>$ true (the default value) avoids computing the bottom local cohomology, and when studying a normal ring, setting AssumeNormal $\Rightarrow>$ true (the default is false) avoids computing the bottom two local cohomologies.

By default the algorithm checks for $F$-injectivity at all points of Spec $R$. However, one can choose to check $F$-injectivity only at the origin by setting the option AtOrigin to true. 


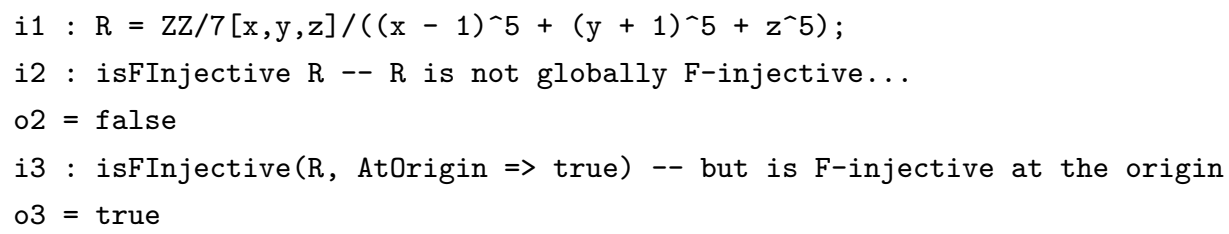

\section{B. F-regularity.}

Definition 3.2 [Hochster and Huneke 1990; Hara and Watanabe 2002]. A ring $R$ is called strongly $F$ regular if the (big) test ideal $\tau(R)$ is equal to $R$. Likewise a pair $\left(R, f^{t}\right)$ is called strongly $F$-regular if $\tau\left(R, f^{t}\right)=R$.

The command isFRegular checks whether a ring or pair is strongly $F$-regular. Below are two examples, one of which is $F$-regular and the other is not.

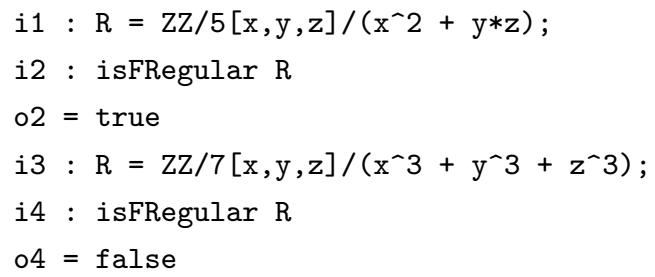

We can also check whether a pair $\left(R, f^{t}\right)$ is $F$-regular.

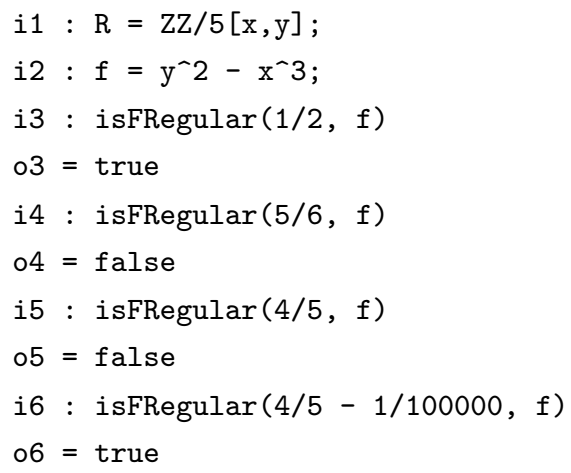

All of these checks are done by actually computing the test ideal, as described in Section 4.

If the input ring is $\mathbb{Q}$-Gorenstein, then in each of the cases above, the output is a boolean indicating if the ring is strongly $F$-regular. If the input ring is not $\mathbb{Q}$-Gorenstein, then the algorithm can be used to determine if a ring is strongly $F$-regular, but cannot prove that a ring is not strongly $F$-regular (this latter functionality can, however, be enabled by setting QGorensteinIndex $\Rightarrow>$ infinity).

In the case that $R$ is $\mathbb{Q}$-Gorenstein, the algorithm works by computing the test ideal $\tau$ of the ring (or the pair) using testIdeal and checking whether $\tau=R$. In the non- $\mathbb{Q}$-Gorenstein case, the algorithm checks for strong $F$-regularity by computing better and better approximations of the test ideal, and checking whether any of these is the unit ideal. To compute approximations of the test ideal, the algorithm computes a test element $c$ with testElement and then uses frobeniusRoot to compute the $e$-th root 
of $c\left(I^{\left[p^{e}\right]}: I\right)$; appropriate modifications are made for pairs. If at any step the approximation is the unit ideal, then the algorithm returns true. Otherwise the algorithm continues checking for each $e$ until a specified limit is reached. The default limit is 2, and can be changed using the option DepthOfSearch.

A number of options can be used to speed up the performance of some of the internal functions. The option AssumeDomain can be used if $R$ is an integral domain, FrobeniusRootStrategy chooses a strategy for internal frobeniusRoot calls, MaxCartierIndex sets the maximum Gorenstein index to search for when working with a $\mathbb{Q}$-Gorenstein ambient ring, and QGorensteinIndex allows the user to specify the $\mathbb{Q}$-Gorenstein index of the ring.

The default behavior of isfRegular is that it checks for strong $F$-regularity globally. If the option AtOrigin is set to true, the algorithm will only check $F$-regularity at the origin, by checking whether the computed test ideal is in the irrelevant ideal. Below are examples for both a ring and a pair.

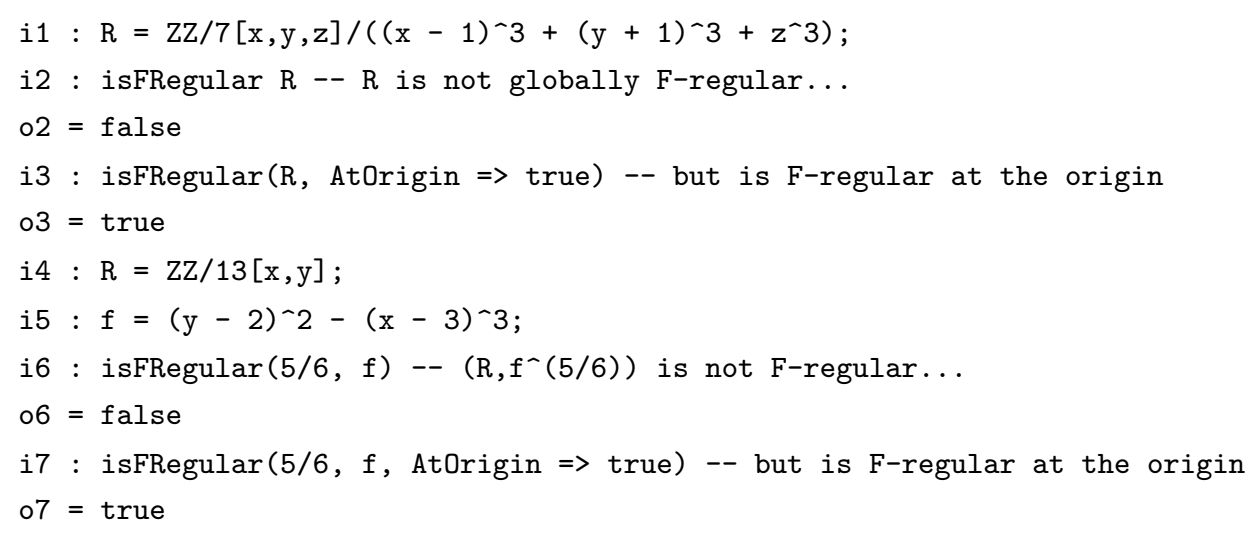

\section{C. F-purity.}

Definition 3.3. A ring $R$ is called $F$-pure if the inclusion $R \hookrightarrow R^{1 / p^{e}}$ is a pure map, i.e., the tensor of this map with any $R$-module remains injective. If $R^{1 / p}$ is a finite $R$-module, this is equivalent to requiring that the inclusion $R \hookrightarrow R^{1 / p}$ split as a map of $R$-modules.

The function isFPure checks whether a ring is $F$-pure. Either a ring or a defining ideal can be input, as seen in the following example.

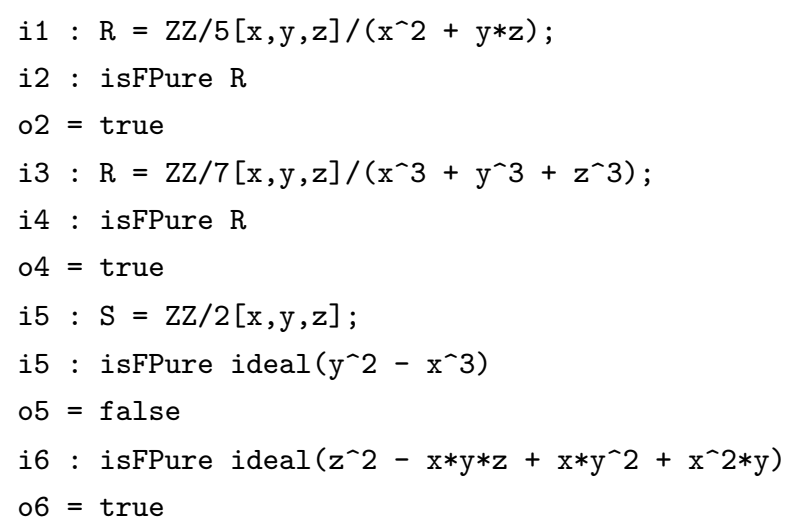


The algorithm works by applying Fedder's criterion [1983], which states that a local ring $(R, \mathfrak{m})$ is $F$-pure if and only if $\left(I^{[p]}: I\right) \nsubseteq \mathfrak{m}^{[p]}$. When AtOrigin is set to true, the algorithm checks $F$-purity only at the origin, by explicitly checking the above containment. When AtOrigin is set to false, which is its default value, the algorithm computes the non $F$-pure locus, by applying frobeniusRoot to $I^{[p]}: I$. If the non $F$-pure locus is the whole ring, the algorithm returns true.

\section{D. F-rationality.}

Definition 3.4. Suppose that $R$ is a Cohen-Macaulay ring and that $T_{R}: \omega_{R^{1 / p}} \rightarrow \omega_{R}$ is the canonical dual to the Frobenius. We say that $R$ has $F$-rational singularities, or simply that $R$ is $F$-rational, if there are no nonzero proper submodules $M$ of $\omega_{R}$ such that $T_{R}\left(M^{1 / p}\right) \subseteq M$.

The command isfRational checks if a ring is $F$-rational.

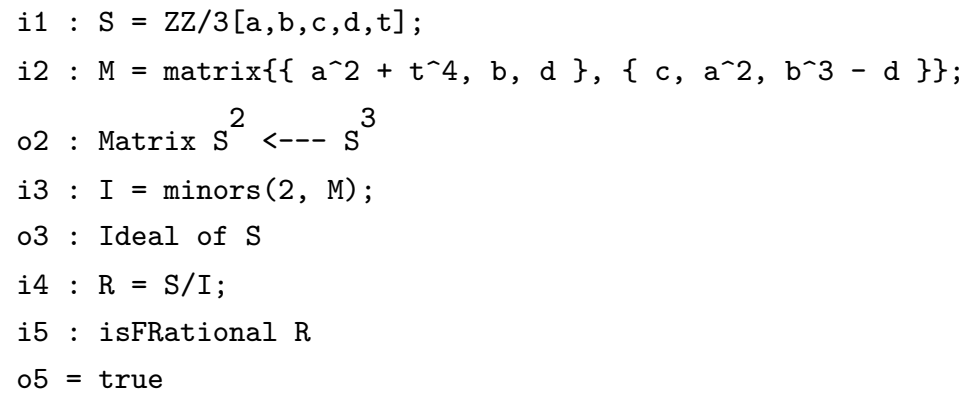

The algorithm used by isFRational first checks if the ring is Cohen-Macaulay, unless the option AssumeCM is set to true. If the ring is not Cohen-Macaulay then false is returned. Next, the algorithm computes the test module $M \subseteq \omega_{R}$ and checks to see if $\omega_{R} \subseteq M$; see the next section for the description of a test module.

The options AssumeDomain and FrobeniusRootStrategy can be used to improve the speed of the testModule computation. By default, these options are set to false and Substitution, respectively. Finally, if AtOrigin is set to true, then $F$-rationality is checked only at the origin.

4. TeSt IDEALS. In this section, we explain how to compute parameter test modules, parameter test ideals, test ideals, and HSLG modules. ${ }^{3}$

4A. Parameter test modules. Given a reduced ring $R$ of finite type over a perfect field $k$, the Frobenius map $R \hookrightarrow R^{1 / p^{e}}$ is dual to

$$
T: \omega_{R^{1 / p^{e}}} \rightarrow \omega_{R}
$$

As in Section 2B, we can represent the canonical module $\omega_{R} \subseteq R$ as an ideal, we can write $R=S / I$, and so we can find an element $u \in S^{1 / p^{e}}$ representing the map $T: \omega_{R^{1 / p^{e}}} \rightarrow \omega_{R}$; see Section 2B or [Katzman 2008].

\footnotetext{
${ }^{3}$ HSLG modules can be used to give a scheme structure to the $F$-injective or $F$-pure locus.
} 
Definition 4.1. The parameter test submodule is the smallest submodule $M \subseteq \omega_{R}$ (and hence ideal of $R$, since $M \subseteq \omega_{R} \subseteq R$ ) that agrees generically with $\omega_{R}$ and that satisfies

$$
T\left(M^{1 / p^{e}}\right) \subseteq M
$$

for some $e>0$ (or equivalently for all $e>0$ ).

Using Macaulay2, we can compute this using the testModule command as follows.

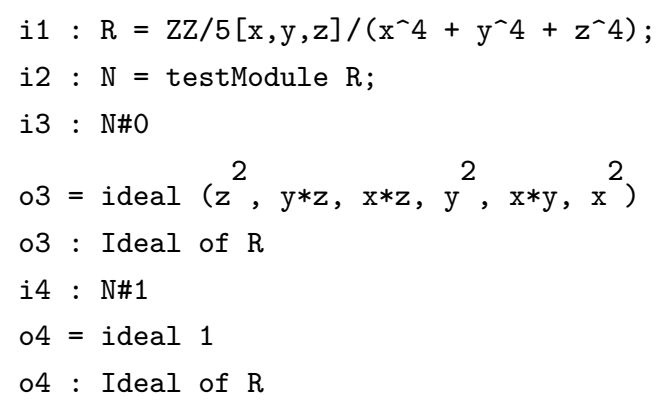

The output of testModule is a sequence, consisting of three items:

(1) The test module itself, given as an ideal of $R$.

(2) The canonical module that contains the test module, given as an ideal of $R$. (Note the representation of the canonical module as an ideal is not unique; it is only unique up to isomorphism, hence it is important to return this module as well).

(3) The element $u$ described above (not displayed above, since it takes a lot of space).

Note since this ring is Gorenstein, the canonical module is simply represented as the unit ideal. Here is another example, where the ring is not Gorenstein.

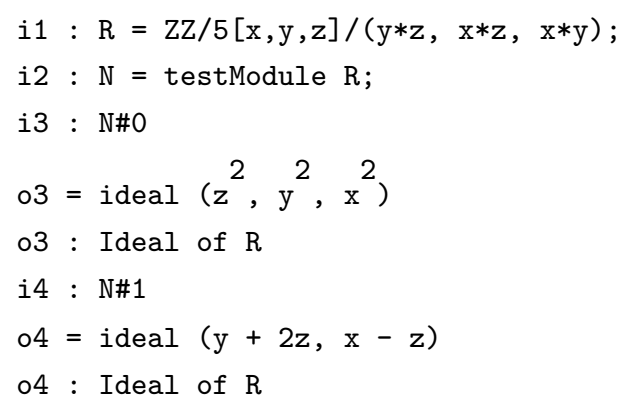

We briefly explain how this is computed: First, we find a test element.

Remark 4.2 (computation of test elements). We recall that, roughly, an element of the Jacobian ideal that is not contained in any minimal prime is a test element [Hochster 2007]. We compute test elements by computing random partial derivatives (and linear combinations thereof) until we find an element that does not vanish at all the minimal primes. This method is much faster than computing the entire 
Jacobian ideal. If it is known that the ring is a domain, setting AssumeDomain $\Rightarrow$ true can speed this up further.

After the test element $c$ has been identified, we pull back the ideal $\omega_{R}$ to an ideal $J \subseteq S$. Next, we compute the following ascending sequence of ideals where $u$ represents $T: \omega_{R^{1 / p^{e}}} \rightarrow \omega_{R}$ as above:

$$
\begin{aligned}
& J_{0}:=c J \\
& J_{1}:=J_{0}+\left(u J_{0}\right)^{[1 / p]} \\
& J_{2}:=J_{1}+\left(u J_{1}\right)^{[1 / p]}=J_{0}+\left(u J_{0}\right)^{[1 / p]}+\left(u^{1+p} J_{0}\right)^{\left[1 / p^{2}\right]} \\
& J_{3}:=J_{2}+\left(u J_{2}\right)^{[1 / p]}=J_{0}+\left(u J_{0}\right)^{[1 / p]}+\left(u^{1+p} J_{0}\right)^{\left[1 / p^{2}\right]}+\left(u^{1+p+p^{2}} J_{0}\right)^{\left[1 / p^{3}\right]}
\end{aligned}
$$

As soon as this ascending sequence of ideals stabilizes, we are done. In fact, because this strategy is used in several contexts, the user can call it directly for a chosen ideal $J$ and $u$ with the function ascendIdeal (this is done for test ideals below).

We can also compute parameter test modules of pairs $\left(\omega_{R}, f^{t}\right)$ with $t \in \mathbb{Q}_{\geq 0}$. This is done by modifying the element $u$ when the denominator of $t$ is not divisible by $p$. When $t$ has $p$ in its denominator, we rely on the fact (see [Blickle et al. 2008; Schwede and Tucker 2014a]) that

$$
\begin{aligned}
T\left(\tau\left(\omega_{R}, f^{a}\right)\right) & =\tau\left(\omega_{R}, f^{a / p}\right), \\
\text { frobeniusRoot }\left(1, \mathrm{u} * \mathrm{I}_{1}\right) & =\mathrm{I}_{2},
\end{aligned}
$$

where the second line roughly explains how this is accomplished internally. Here $I_{1}$ is $\tau\left(\omega_{R}, f^{a}\right)$ pulled back to $S$ and, likewise, $I_{2}$ defines $\tau\left(\omega_{R}, f^{a / p}\right)$ modulo the defining ideal of $R$.

Remark 4.3 (optimizations in ascendIdeal and other testModule computations). Throughout the computations described above, we very frequently use the following fact:

$$
\left(f^{p} \cdot J\right)^{[1 / p]}=f \cdot\left(J^{[1 / p]}\right) .
$$

To access this enhancement, one should try to pass functions like ascendIdeal and frobeniusRoot the elements and their exponents (see the documentation). In particular, when computing the $p^{e}$-th Frobenius root of an ideal of the form $f^{n} \cdot J$, one should not multiply out $f^{n}$ and $J$, nor even raise $f$ to the $n$-th power, but rather call frobeniusRoot (e, $n, f, J)$.

4B. Parameter test ideals. The parameter test ideal is simply the annihilator of $\omega_{R} / \tau\left(\omega_{R}\right)$. In other words, it is

$$
\left(\tau\left(\omega_{R}\right): \omega_{R}\right)
$$

This can also be described as

$$
\bigcap_{I}\left(I^{*}: I\right)
$$

where $I$ varies over ideals defined by a partial system of parameters, and $I^{*}$ denotes its tight closure. This latter description is not computable, however. 
Example 4.4. In this example we repeat the calculation in [Katzman 2008, §9].

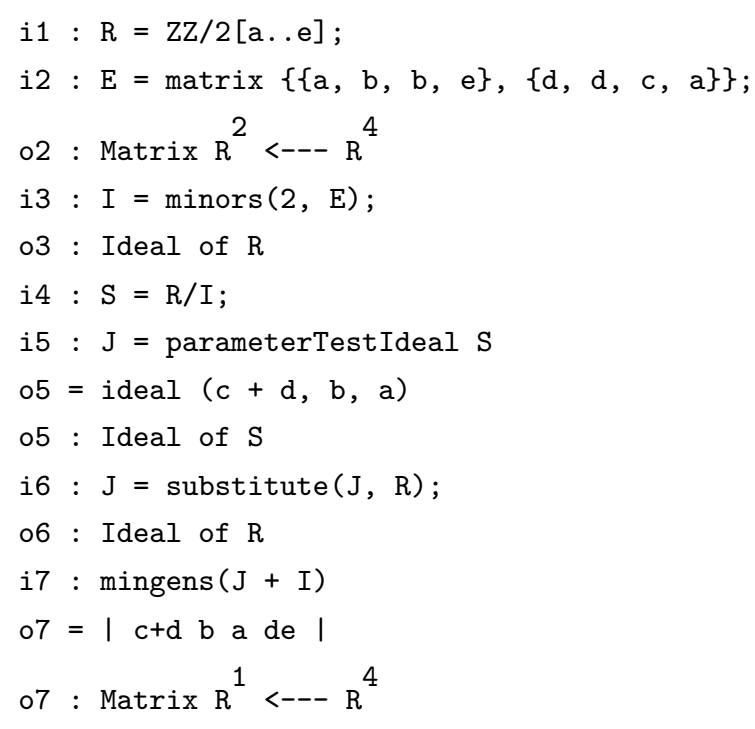

4C. Test ideals. For an $F$-finite reduced ring $R=S / I$, where $S$ is a regular ring, the (big) test ideal ${ }^{4}$ of $R$ is the smallest ideal $J$ in $R$, not contained in any minimal prime, such that for all $e>0$ and all $\phi \in \operatorname{Hom}_{R}\left(R^{1 / p^{e}}, R\right)$, we have

$$
\phi\left(J^{1 / p^{e}}\right) \subseteq J
$$

In the case that $R$ is Gorenstein, $\operatorname{Hom}_{R}\left(R^{1 / p^{e}}, R\right)$ is a cyclic $R^{1 / p^{e}}$-module generated by $\Phi_{e}$, which corresponds with the map $T$ above based on the identification $\omega_{R} \cong R$ [Blickle and Schwede 2013]. More generally, if $R$ is $\mathbb{Q}$-Gorenstein with index not divisible by $p$, then for at least sufficiently divisible $e>0$, such a generating $\Phi_{e}$ still exists.

If such a $\Phi_{e}$ exists, it can be identified to a generator of the module

$$
\left(I^{\left[p^{e}\right]}: I\right) / I^{\left[p^{e}\right]}
$$

by Fedder's lemma [1983]. Hence we can find a corresponding ${ }^{5} u \in I^{\left[p^{e}\right]}: I$. In this case, if $c \in S$ is the preimage of a test element of $R$, then setting $I_{0}=c R$, it follows that

$$
\tau(R)=\operatorname{ascendIdeal}\left(\mathrm{e}, \mathrm{u}, \mathrm{I}_{0}\right),
$$

where ascendIdeal is the method explained above, in (4-1). The $e$ here means all Frobenius roots are taken as multiples of $e$. In other words, we first compute $\mathrm{I}_{0}+\left(\mathrm{u} \cdot \mathrm{I}_{0}\right)^{1 / \mathrm{p}^{[e]}}$. Then we compute

$$
I_{0}+\left(u \cdot I_{0}\right)^{1 / p^{[e]}}+\left(u^{p^{e}+1} \cdot I_{0}\right)^{1 / p^{[2 e]}}
$$

etc.

\footnotetext{
${ }^{4}$ The notion of test ideals was originally introduced in [Hochster and Huneke 1990] in the context of tight closure in finitely generated modules, whereas our notion of test ideals arises from tight closure in ("big") modules which are possibly not finitely generated. Confusingly, big test ideals are included in (small) test ideals.

${ }^{5}$ This is done via the function QGorensteinGenerator.
} 
Here is an example (a $\mathbb{Z} / 3 \mathbb{Z}$-quotient, where $3 \mid(7-1)$ ), where exactly this logic occurs.

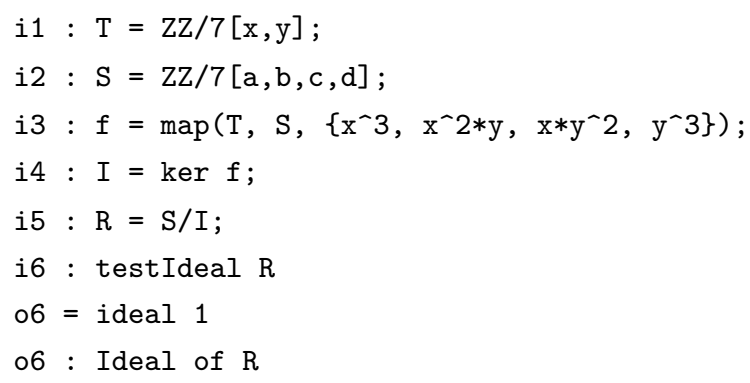

However, the term $u$ constructed above can be quite complicated if $e>1$ (which happens exactly when $(p-1) K_{R}$ is not Cartier). For instance, even in the above example we have an extremely complex $u$ :

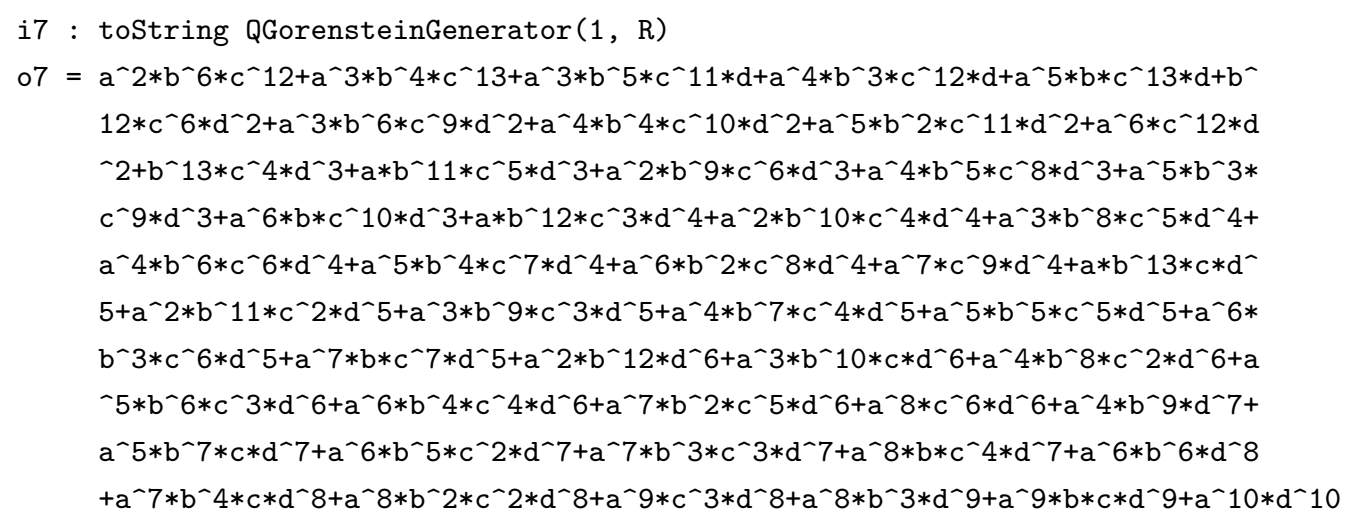

Therefore, we use a different strategy if either $(p-1) K_{R}$ is not Cartier or, more generally, if $R$ is $\mathbb{Q}$-Gorenstein of index divisible by $p$. In these situations, this alternate strategy typically appears to be faster. We rely on the observation (see [Blickle et al. 2015]) that

$$
\tau\left(\omega_{R}, K_{R}\right) \cong \tau(R) .
$$

In fact, by embedding $\omega_{R} \subseteq R$, we can compute $g$ so that $\tau\left(\omega_{R}, K_{R}\right)=g \cdot \tau(R)$. We can therefore find $\tau(R)$ if we can find $\tau\left(\omega_{R}, K_{R}\right)$. Next, if $K_{R}$ is $\mathbb{Q}$-Cartier with $n K_{R}=\operatorname{div}_{R}(f)$ for some $f \in R$ and $n>0$, then

$$
\tau\left(\omega_{R}, K_{R}\right)=\tau\left(\omega_{R}, f^{1 / n}\right) .
$$

Thus we directly compute $\tau\left(\omega_{R}, f^{1 / n}\right)$ via the command testModule $(1 / \mathrm{n}, \mathrm{f})$. Consider the following example, a $\mu_{3}$-quotient, which uses the logic described above.

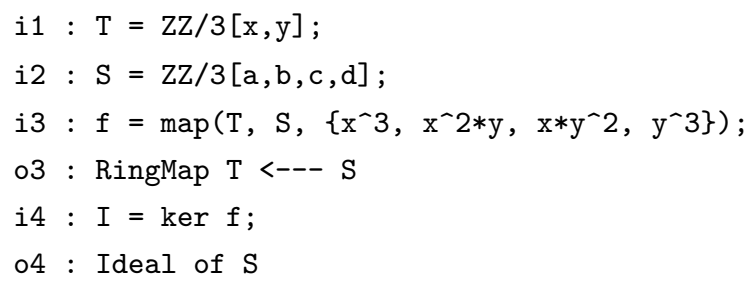




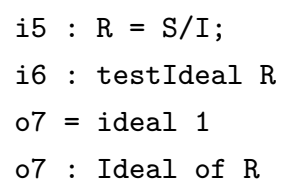

Remark 4.5 (nongraded caveats). It frequently happens that $\left(I^{\left[p^{e}\right]}: I\right) / I^{\left[p^{e}\right]}$ is principal but Macaulay 2 cannot identify the principal generator (since Macaulay 2 cannot always find minimal generators of nongraded ideals or modules). The same thing can happen when computing the element $u$ corresponding to the map $T: \omega_{R^{1 / p}} \rightarrow \omega_{R}$, as described in Section 2B. In such situations, instead of a single $u$, Macaulay2 will produce $u_{1}, \ldots, u_{n}$ (all multiples of $u$, and $u$ is a linear combination of the $u_{i}$ ). Instead of computing the ideal

$$
(u \cdot J)^{[1 / p]},
$$

we compute

$$
\left(u_{1} \cdot J\right)^{[1 / p]}+\cdots+\left(u_{n} \cdot J\right)^{[1 / p]},
$$

which will produce the same answer.

We can similarly use the testIdeal command to compute test ideals of pairs, $\tau\left(R, f^{t}\right)$, and even mixed test ideals, $\tau\left(R, f_{1}^{t_{1}} \cdots f_{n}^{t_{n}}\right)$.

4D. HSLG module; computing F-pure submodules of rank-1 Cartier modules. Again, take the maps $T^{e}: \omega_{R^{1 / p^{e}}} \rightarrow \omega_{R}$ we have considered throughout this section. From [Hartshorne and Speiser 1977; Lyubeznik 1997; Gabber 2004], we have the theorem that the descending images

$$
\omega_{R} \supseteq T\left(\omega_{R^{1 / p}}\right) \supseteq \cdots \supseteq T^{e}\left(\omega_{R^{1 / p^{e}}}\right) \supseteq T^{e+1}\left(\omega_{R^{1 / p^{e+1}}}\right) \supseteq \cdots
$$

stabilize for $e \gg 0$. The function FPureModule computes the stable submodule in this chain, called the HSLG module, and returns a sequence containing the following items:

(1) The HSLG module.

(2) The canonical module, embedded (nonuniquely) as an ideal.

(3) The element $u$ representing the map on the canonical module (see Section 2B).

(4) The value of $e>0$ at which the descending images in (4-2) stabilize. This is sometimes also called the HSLG number of the canonical module as a Cartier module.

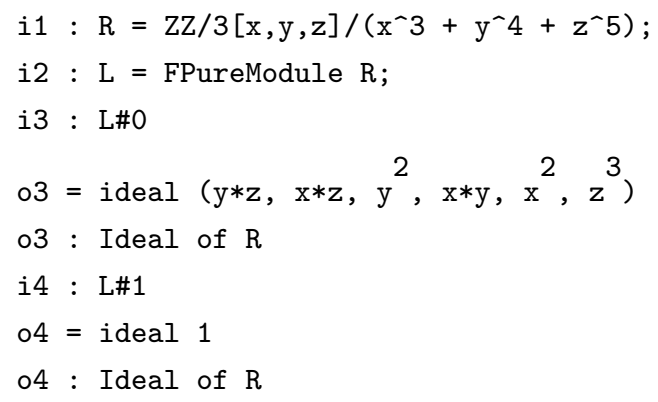




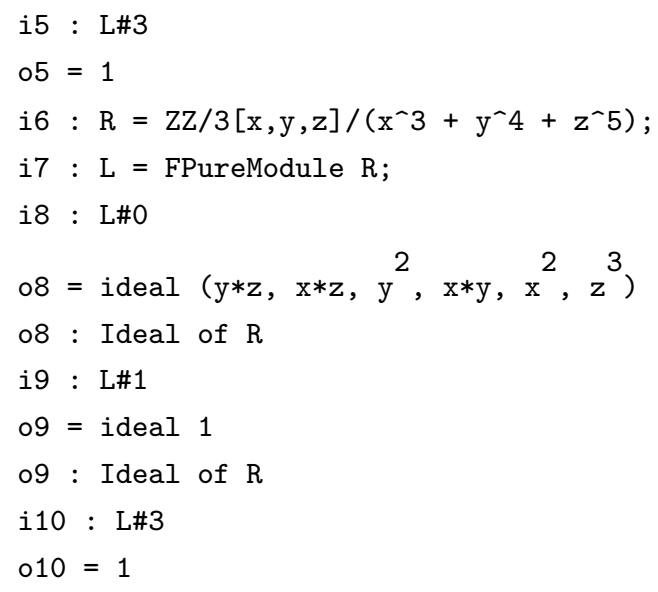

More generally, for any ideal $J$ with a map $\phi: J^{1 / p^{e}} \rightarrow J$, we have that the images

$$
J \supseteq \phi\left(J^{1 / p^{e}}\right) \supseteq \phi^{2}\left(J^{1 / p^{2 e}}\right) \supseteq \cdots
$$

stabilize as well (in fact, the analogous result even holds for modules [Gabber 2004]).

4E. Computing the level of a polynomial. Another interesting invariant that can be calculated using this package is the so-called level of a polynomial; more precisely:

Definition 4.6. Let $K$ be an $F$-finite field of prime characteristic $p$, and let $f \in R=K\left[x_{1}, \ldots, x_{d}\right]$. We define the level of $f$ as the smallest possible integer $e$ where the descending chain

$$
R=\left(f^{p^{0}-1}\right)^{\left[1 / p^{0}\right]} \supseteq\left(f^{p-1}\right)^{[1 / p]} \supseteq\left(f^{p^{2}-1}\right)^{\left[1 / p^{2}\right]} \supseteq \cdots \supseteq\left(f^{p^{i}-1}\right)^{\left[1 / p^{i}\right]} \supseteq \cdots
$$

stabilizes.

This invariant was introduced in [Alvarez-Montaner et al. 2005]. It is also essentially the same data as the HSLG number of the pair $\left(R, f^{1}\right)$ as computed in (4) above (it is that number +1$)$. One interesting particular case is when $K=\mathbb{F}_{p}$ and $f$ is the defining homogeneous polynomial of a hyperelliptic curve of genus $g$; when $g=1$, it was proved in [Boix et al. 2015] that the corresponding elliptic curve defined by $f$ is ordinary if and only if its level is 1, (equivalently, if and only if $(R /(f))_{(x, y, z)}$ is $F$-pure, ) and supersingular if and only if its level is 2 . When the genus is at least 2, level 2 is a necessary (but not sufficient) condition for the curve for being ordinary [Blanco-Chacón et al. 2018]; in this case, one also has that, if the curve is supersingular, then its level has to be at least 3, so the level can always distinguish these two properties in any genus. We illustrate these results by means of the following examples.

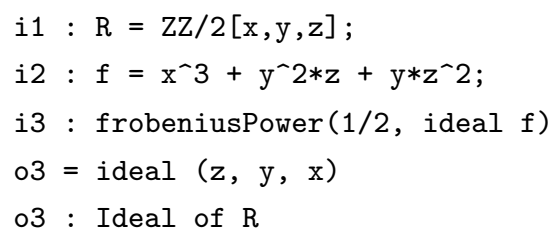




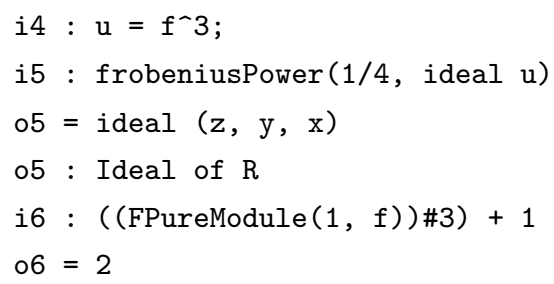

This shows that this elliptic curve has level 2; hence it is supersingular. The next example shows a nonordinary hyperelliptic curve of genus 2 with level 2.

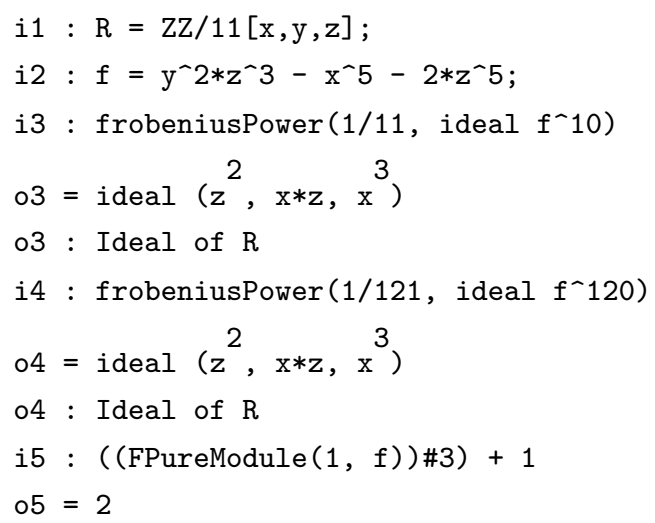

5. IdeAls COMPATIBLE With A GiVen $p^{-e}$-Linear MAP. Throughout this section, let $R$ denote a polynomial $\mathbb{K}\left[x_{1}, \ldots, x_{n}\right]$. In this section we address the following question: given an $R$-linear map $\phi: R^{1 / p^{e}} \rightarrow R$, what are the ideals $I \subseteq R$ such that $\phi\left(I^{1 / p^{e}}\right) \subseteq I$ ? We call these ideals $\phi$-compatible.

Recall that $\operatorname{Hom}_{R}\left(R^{1 / p^{e}}, R\right)$ is a principal $R^{1 / p^{e}}$-module generated by a trace map $T \in \operatorname{Hom}_{R}\left(R^{1 / p^{e}}, R\right)$, constructed as in Section 2A (see [Fedder 1983, Lemma 1.6] and [Brion and Kumar 2005, Example 1.3.1]).

We can now write our given $\phi$ as multiplication by some $u^{1 / p^{e}}$ followed by $T$ and it is not hard to see that an ideal $I \subseteq R$ is $\phi$-compatible if and only if $u I \subseteq I^{\left[p^{e}\right]}$.

Theorem 5.1. If $\phi$ is surjective, there are finitely many $\phi$-compatible ideals, consisting of all possible intersections of $\phi$-compatible prime ideals (see [Kumar and Mehta 2009, Schwede 2009, Sharp 2007, Enescu and Hochster 2008]). In general, there are finitely many $\phi$-compatible prime ideals not containing $\sqrt{\operatorname{Image} \phi}$ (see [Katzman and Schwede 2012]).

The method compatibleIdeals produces the finite set of $\phi$-compatible prime ideals in the second claim of Theorem 5.1.

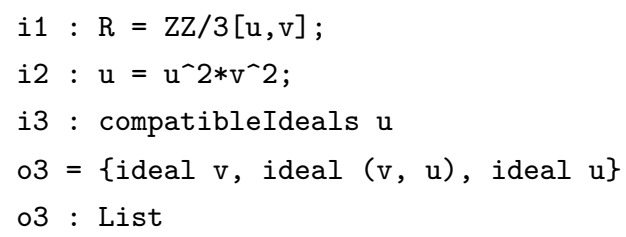


The defining condition $u I \subseteq I^{\left[p^{e}\right]}$ for $\phi$-compatible ideals allows us to think of these in a dual form: write $\mathfrak{m}=\left(x_{1}, \ldots, x_{n}\right), E=E_{R_{\mathfrak{m}}}\left(R_{\mathfrak{m}} / \mathfrak{m}\right)=H_{\mathfrak{m}}^{n}(R)$, and let $\Theta: E \rightarrow E$ be the $p^{e}$-linear map ${ }^{6}$ induced from the Frobenius map on $R$. If $\psi=u \Theta$, then $\psi \operatorname{Ann}_{E} I \subseteq \operatorname{Ann}_{E} I$ if and only if $u I \subseteq I^{\left[p^{e}\right]}$. Thus finding all $R$-submodules of $E$ compatible with $\psi=u \Theta$ also amounts to finding all $\phi$-compatible ideals, where $\phi=T \circ u^{1 / p^{e}}$.

Example 5.2. We return to Example 4.4. In [Katzman 2008, §9] it is shown that there is a surjection $\operatorname{Ann}_{E} I \rightarrow H_{\mathfrak{m}}^{2}(R / I)$ which is compatible with the induced $p^{1}$-linear map on $H_{\mathfrak{m}}^{2}(R / I)$ and the $p^{1}$-linear map $u \Theta$ on $\operatorname{Ann}_{E} I$, where $u$ is computed as follows.

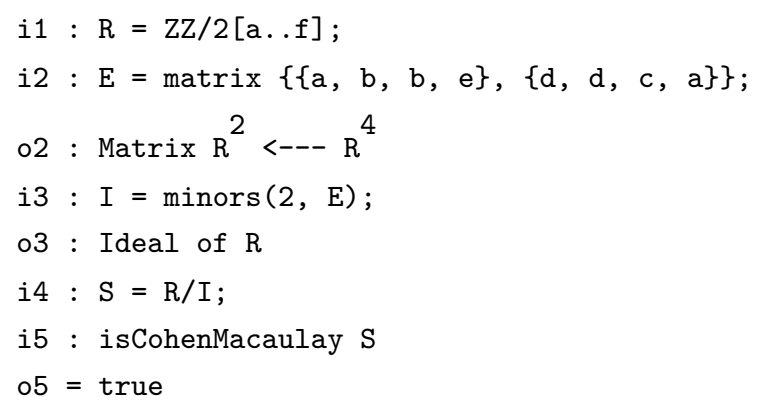

In [Katzman 2008] it is shown that since $R / I$ is Cohen-Macaulay, $u$ can be taken as the generator of the cyclic module $\left(I^{[p]}: I\right) \cap\left(\Omega^{[p]}: \Omega\right) / I^{[p]}$ where $\Omega \subseteq R$ is an ideal whose image in $R / I$ is a canonical module of that ring.

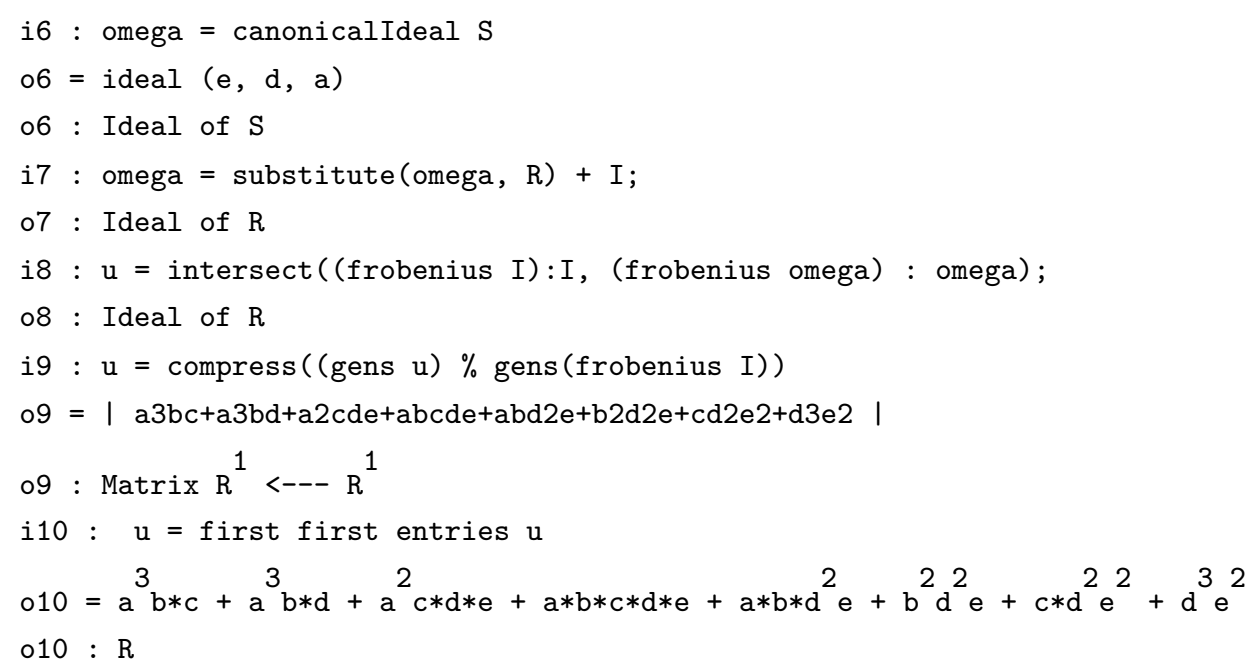

Now we can compute all annihilators of $R$-submodules of $E$ stable under the $p^{1}$-linear map $u T$.

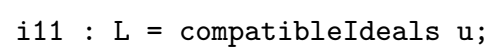

${ }^{6}$ That is, $\Theta$ is additivive and $\Theta(r a)=r p^{e} \Theta(a)$ for all $a \in E$ and $r \in R$. 


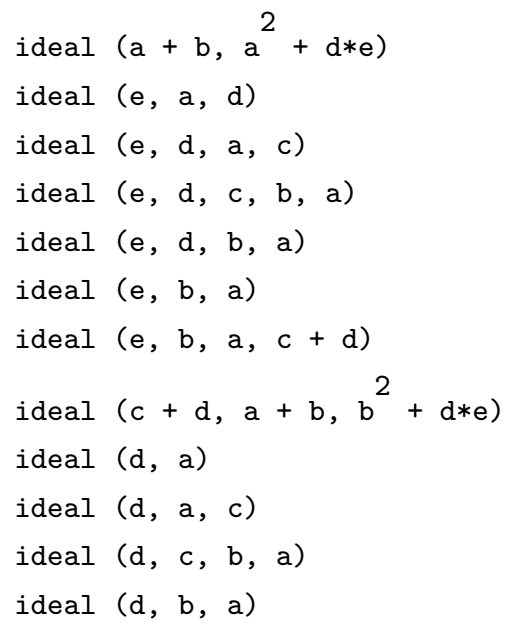

We can also compute all annihilators of $R$-submodules of $H_{\mathfrak{m}}^{2}(R / I)$ stable under the $p^{1}$-linear map induced from the Frobenius map on $R / I$.

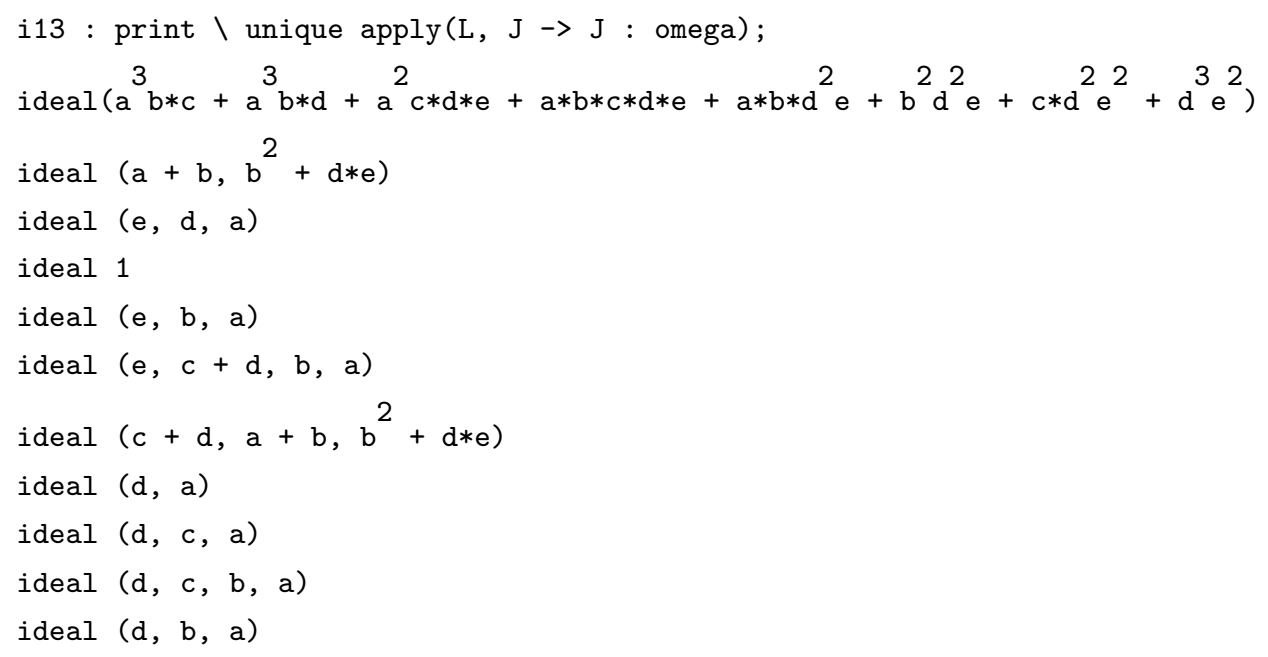

6. Future Plans. In [Katzman and Zhang 2014], the algorithms behind the methods in Section 5 were extended to compute prime annihilators of submodules of Artinian modules compatible with a given $p^{e}$-linear map. This would require, among other things, a faster implementation of our method for finding Frobenius roots of submodules of free modules.

On the other hand, it should be possible to compute test ideals of pairs $\left(R, \mathfrak{a}^{t}\right)$ even when $\mathfrak{a}$ is not principal. One strategy to do this is outlined in [Schwede and Tucker 2014b] although certain improvements can be made.

We hope to achieve all these things during a future Macaulay2 workshop. We also want to bring to the reader's attention the package FrobeniusThresholds, which computes $F$-pure thresholds, $F$-thresholds, $F$-jumping numbers and more! 
ACKNOWLEDGEMENTS. The authors thank other contributors of the TestIdeals package, namely Erin Bela, Juliette Bruce, Drew Ellingson, Matthew Mastroeni, and Maral Mostafazadehfard. We also thank the referees for numerous useful comments on the package and on this paper, and Thomas Polstra for pointing out typos in a previous version of the paper. This paper, and the finishing touches to the TestIdeals package, were made at the University of Utah in 2018, and the visiting authors thank the Department of Mathematics for its hospitality. The authors also thank the referees for their numerous useful comments on both this paper and the code. Additional work on the package was done at the Institute of Mathematics and its Applications, and the authors are grateful for its generous support for a 2019 Coding Sprint on the FrobeniusThresholds package.

SUPPLEMENT. The online supplement contains version 1.01 of the TestIdeals package.

\section{REFERENCES.}

[Alvarez-Montaner et al. 2005] J. Alvarez-Montaner, M. Blickle, and G. Lyubeznik, "Generators of $D$-modules in positive characteristic", Math. Res. Lett. 12:4 (2005), 459-473. MR Zbl

[Blanco-Chacón et al. 2018] I. Blanco-Chacón, A. F. Boix, S. Fordham, and E. S. Yilmaz, "Differential operators and hyperelliptic curves over finite fields", Finite Fields Appl. 51 (2018), 351-370. MR Zbl

[Blickle and Schwede 2013] M. Blickle and K. Schwede, " $p^{-1}$-linear maps in algebra and geometry”, pp. 123-205 in Commutative algebra, edited by I. Peeva, Springer, 2013. MR Z Zbl

[Blickle et al. 2008] M. Blickle, M. Mustaţă, and K. E. Smith, "Discreteness and rationality of F-thresholds", Michigan Math. J. 57 (2008), 43-61. MR Zbl

[Blickle et al. 2009] M. Blickle, M. Mustaţă, and K. E. Smith, "F-thresholds of hypersurfaces”, Trans. Amer. Math. Soc. 361:12 (2009), 6549-6565. MR Zbl

[Blickle et al. 2010] M. Blickle, K. Schwede, S. Takagi, and W. Zhang, "Discreteness and rationality of $F$-jumping numbers on singular varieties", Math. Ann. 347:4 (2010), 917-949. MR Zbl

[Blickle et al. 2015] M. Blickle, K. Schwede, and K. Tucker, “F-singularities via alterations”, Amer. J. Math. 137:1 (2015), 61-109. MR Zbl

[Boix et al. 2015] A. F. Boix, A. De Stefani, and D. Vanzo, "An algorithm for constructing certain differential operators in positive characteristic", Matematiche (Catania) 70:1 (2015), 239-271. MR

[Brion and Kumar 2005] M. Brion and S. Kumar, Frobenius splitting methods in geometry and representation theory, Progress in Mathematics 231, Birkhäuser, Boston, 2005. MR Zbl

[Enescu and Hochster 2008] F. Enescu and M. Hochster, "The Frobenius structure of local cohomology", Algebra Number Theory 2:7 (2008), 721-754. MR Zbl

[Fedder 1983] R. Fedder, "F-purity and rational singularity”, Trans. Amer. Math. Soc. 278:2 (1983), 461-480. MR Zbl

[FrobeniusThresholds] J. Bruce, D. Hernández, K. Schwede, D. Smolkin, P. Teixeira, and E. Witt, "FrobeniusThresholds: A package for calculations of F-thresholds.", Macaulay2 package Version 2.0, available at https://github.com/Macaulay2/M2/ tree/master/M2/Macaulay2/packages.

[Gabber 2004] O. Gabber, "Notes on some $t$-structures", pp. 711-734 in Geometric aspects of Dwork theory, edited by A. Adolphson et al., Walter de Gruyter, Berlin, 2004. MR Zbl

[Hara and Watanabe 2002] N. Hara and K.-I. Watanabe, "F-regular and F-pure rings vs. log terminal and log canonical singularities", J. Algebraic Geom. 11:2 (2002), 363-392. MR Zbl

[Hartshorne and Speiser 1977] R. Hartshorne and R. Speiser, "Local cohomological dimension in characteristic p", Ann. of Math. (2) 105:1 (1977), 45-79. MR Zbl 
[Hernández 2014] D. J. Hernández, "F-pure thresholds of binomial hypersurfaces”, Proc. Amer. Math. Soc. 142:7 (2014), 2227-2242. MR Zbl

[Hernández 2015] D. J. Hernández, “F-invariants of diagonal hypersurfaces”, Proc. Amer. Math. Soc. 143:1 (2015), 87-104. MR Zbl

[Hernández and Teixeira 2017] D. J. Hernández and P. Teixeira, " $F$-threshold functions: syzygy gap fractals and the twovariable homogeneous case", J. Symbolic Comput. 80:part 2 (2017), 451-483. MR Zbl

[Hernández et al. 2018] D. J. Hernández, P. Teixeira, and E. E. Witt, "Frobenius powers", 2018. arXiv

[Hernández et al. 2019] D. J. Hernández, K. Schwede, P. Teixeira, and E. E. Witt, "The FrobeniusThresholds package for Macaulay2", preprint, 2019. arXiv

[Hernández et al. 2020] D. J. Hernández, P. Teixeira, and E. E. Witt, "Frobenius powers of some monomial ideals", J. Pure Appl. Algebra 224:1 (2020), 66-85. MR Zbl

[Hochster 2007] M. Hochster, "Foundations of tight closure theory", lecture notes, University of Michigan, 2007, available at http://www.math.lsa.umich.edu/ hochster/711F07/fndtc.pdf.

[Hochster and Huneke 1990] M. Hochster and C. Huneke, "Tight closure, invariant theory, and the Briançon-Skoda theorem", J. Amer. Math. Soc. 3:1 (1990), 31-116. MR Zbl

[Katzman 2008] M. Katzman, "Parameter-test-ideals of Cohen-Macaulay rings", Compos. Math. 144:4 (2008), 933-948. MR $\mathrm{Zbl}$

[Katzman 2010] M. Katzman, "Frobenius maps on injective hulls and their applications to tight closure", J. Lond. Math. Soc. (2) 81:3 (2010), 589-607. MR Zbl

[Katzman and Schwede 2012] M. Katzman and K. Schwede, "An algorithm for computing compatibly Frobenius split subvarieties”, J. Symbolic Comput. 47:8 (2012), 996-1008. MR Zbl

[Katzman and Zhang 2014] M. Katzman and W. Zhang, "Annihilators of Artinian modules compatible with a Frobenius map", J. Symbolic Comput. 60 (2014), 29-46. MR Zbl

[Katzman et al. 2009] M. Katzman, G. Lyubeznik, and W. Zhang, "On the discreteness and rationality of $F$-jumping coefficients", J. Algebra 322:9 (2009), 3238-3247. MR Zbl

[Kumar and Mehta 2009] S. Kumar and V. B. Mehta, "Finiteness of the number of compatibly split subvarieties", Int. Math. Res. Not. 2009:19 (2009), 3595-3597. MR Zbl

[Kunz 1969] E. Kunz, "Characterizations of regular local rings of characteristic p", Amer. J. Math. 91 (1969), 772-784. MR $\mathrm{Zbl}$

[Lyubeznik 1997] G. Lyubeznik, " $F$-modules: applications to local cohomology and $D$-modules in characteristic $p>0$ ", J. Reine Angew. Math. 491 (1997), 65-130. MR

[Macaulay2] D. R. Grayson and M. E. Stillman, "Macaulay2: a software system for research in algebraic geometry", available at http://www.math.uiuc.edu/Macaulay2.

[PushForward] C. Raicu, "PushForward: push forwards of finite ring maps", Macaulay2 package, version 0.1, available at https://github.com/Macaulay2/M2/tree/master/M2/Macaulay2/packages.

[Schwede 2009] K. Schwede, “ $F$-adjunction”, Algebra Number Theory 3:8 (2009), 907-950. MR Zbl

[Schwede and Tucker 2012] K. Schwede and K. Tucker, "A survey of test ideals", pp. 39-99 in Progress in commutative algebra 2, edited by C. Francisco et al., Walter de Gruyter, Berlin, 2012. MR Zbl

[Schwede and Tucker 2014a] K. Schwede and K. Tucker, "On the behavior of test ideals under finite morphisms", J. Algebraic Geom. 23:3 (2014), 399-443. MR Zbl

[Schwede and Tucker 2014b] K. Schwede and K. Tucker, "Test ideals of non-principal ideals: computations, jumping numbers, alterations and division theorems", J. Math. Pures Appl. (9) 102:5 (2014), 891-929. MR Zbl

[Sharp 2007] R. Y. Sharp, "Graded annihilators of modules over the Frobenius skew polynomial ring, and tight closure", Trans. Amer. Math. Soc. 359:9 (2007), 4237-4258. MR Zbl 


\section{Alberto F. Boix:}

fernanal@post.bgu.ac.il

Department of Mathematics, Ben-Gurion University of the Negev, Beer-Sheva, Israel

DANIEL J. HERNÁNDEZ:

hernandez@ku.edu

Department of Mathematics, University of Kansas, Lawrence, KS, United States

\section{ZHIBEK KADYRSIZOVA:}

zhibek.kadyrsizova@nu.edu.kz

School of Science and Technology, Nazarbayev University, Astana, Kazakhstan

\section{Mordechai KATZMAN:}

m.katzman@sheffield.ac.uk

Department of Pure Mathematics, University of Sheffield, Sheffield, United Kingdom

\section{SARA MALec:}

malec@hood.edu

Department of Mathematics, Hood College, Frederick, MD, United States

\section{MARCUS ROBINSON:}

robinson@math.utah.edu

Department of Mathematics, University of Utah, Salt Lake City, UT, United States

\section{KARL SCHWEDE:}

schwede@math.utah.edu

Department of Mathematics, University of Utah, Salt Lake City, UT, United States

\section{DANIEL SMOLKIN:}

smolkin@math.utah.edu

Department of Mathematics, University of Utah, Salt Lake City, UT, United States

\section{PEDRo TEIXEIRA:}

pteixeir@knox.edu

Department of Mathematics, Knox College, Galesburg, IL, United States

EMILY E. WITT:

witt@ku.edu

Department of Mathematics, University of Kansas, Lawrence, KS, United States 